\title{
DIVERSITY, DISTRIBUTION AND CONSERVATION OF FISHES IN LAKES OF POKHARA VALLEY, NEPAL
}

\author{
Ganesh Timilsina ${ }^{1}$, Subhash C. Bastola ${ }^{2}$, Sherman Gurung ${ }^{3}$, Kishor K. Pokharel ${ }^{4}$ \\ ${ }^{1-4}$ Department of Zoology, Prithvi Narayan Campus, (TU), Pokhara, Nepal. \\ E-mail: ganeshtimilsina2012@gmail.com
}

\begin{abstract}
Present study deals with fish diversity along with management aspects of lakes in Pokhara Valley. It was conducted during September 2009 to February 2010. Monthly fish sampling was done using gill net with the help of experienced fishermen. Altogether 34 species of fishes were recorded in the present study. The population status of important fish species viz., Tor Tor (HamiltonBuchanan) was found to be endangered, that of Tor putitora (Hamilton-Buchanan), Neolissocheilus hexagonolepis (Mc Clelland), Chagunius chagunio (Hamilton-Buchanan), and Brachydanio rerio (Hamilton-Buchanan) were found to be vulnerable (VU); five species were uncommon (UN) and 24 species were common $(\mathrm{C})$ including common exotic $\left(\mathrm{C}^{*}\right)$. The lakes were found to be influenced by human activities such as direct disposal of domestic sewage, unscientific agricultural practices, construction of dams without fish ladder or passes, deforestation causing soil erosion etc., which were creating threat to the lentic ecosystem.
\end{abstract}

Key words : Lentic ecosystem, deforestation, bioresources, alien species, migration.

\section{INTRODUCTION}

Pokhara valley has nine lakes with rich fish diversity along with other aquatic bioresources. Wetlands are considered to be one of the world's most productive and important natural ecosystems. They are the cradle of aquatic biodiversity and provide the water and primary productivity upon which countless species of plants and animals depend for survival (Dugan, 1990). Properly managed wetlands can be a critical life supporting habitats for millions of people throughout the world (Russel, 1993). Fish constitute the important group of aquatic biodiversity which has been used as food from the time immemorial. The deforestation or denudation of the watersheds or riparian vegetation was mentioned to be an important factor causing landslide, soil erosion, and siltation resulting in degradation of habitat and alteration in run-off which adversely influence the biodiversity, shrinkage of breeding grounds and multiplication of fish population (Wetzel, 2001; Shrestha 2003; Pokharel, 2010a). The published literature on fish biodiversity of Pokhara Valley includes those of Ferrow and Badgami (1980); Agriculture Research Centre Pokhara (Fisheries) (2001-02); Pokharel (1999a), Pokharel et al. (2010), Bista, Dhakal and Gurung (2001/02), which studied on fish diversity and fisheries resources of the water bodies in the valley.

Present study aimed to explore occurrence, distribution and status of fish diversity in major lakes of Pokhara valley. The findings of this study may help for planning in management of lakes. 
Diversity, distribution and conservation of ...

\section{MATERIALS AND METHODS}

\section{Study Area}

Pokhara, a beautiful valley in the western part of Nepal extends between $25^{\circ} 07^{\prime} \mathrm{N}$ to $28^{\circ} 10^{\prime}$ latitude, and $83^{\circ} 50^{\prime} \mathrm{E}$ to $84^{\circ} 50^{\prime} \mathrm{E}$ longitude and lies $800 \mathrm{~m}$ above sea level (Tripathi, 1984-85). The valley has many fascinating water bodies such as lakes, rivers, streams, ponds, creeks, falls etc with rich bioresources. The lakes included in the present study were Phewa, Begnas, Rupa, Maidi and Dipang (Figure 1). Phewa is the biggest lake situated at an altitude of $742 \mathrm{~m}$ asl, covering an area of 5.23 sq.m, extended north to south $6.4 \mathrm{~km}$, east-west width ranging from $50 \mathrm{~m}$ to $2 \mathrm{~km}$ and up to $19 \mathrm{~m}$ in depth. The lake has two perennial creeks i.e., Harpan Khola and Andheri Khola as the main feeding sources. Pardi Khola is the only outlet of the lake which flows downwards to join Phusre Khola. Begnas is the second biggest lake situated at $650 \mathrm{~m}$ asl in the eastern part of the valley. It covers $3.28 \mathrm{sq}$ $\mathrm{km}$ in area and upto $7.5 \mathrm{~m}$ in depth. The main feeding source of the lake is Syankhudi Khola at the upper part of the lake, while Khudi Khola is the natural outlet which meets the Kotre Khola downwards, which finally joins the Seti Gandaki River. Rupa Lake is the third major lake of the Valley, situated at $600 \mathrm{~m}$ asl in the eastern part. It covers $1.15 \mathrm{sqkm}$ in area, being $2.4 \mathrm{~km}$ in length, $0.8 \mathrm{~km}$ in width and $5.0 \mathrm{~m}$ or more in depth. A small perennial creek, Talbesi Khola is the main feeding source of the lake. Tal Khola is the outlet, joins Kotre Khola downwards, which finally meets the Seti Gandaki River near Kotre. Lake Maidi and Dipang are small lakes also situated in the eastern part of the valley. Maidi is $0.53 \mathrm{sqkm}$ and Dipang is $0.32 \mathrm{sqkm}$ in area and are upto $2.5 \mathrm{~m}$ in depth. The lake has its major part covered by thick layer of grass or macrovegetation surrounded by marshy area. Lake Dipang was also having swampy shore with thick aquatic macrophytes most of which has been removed by the lake conservation authorities with participation of local people.

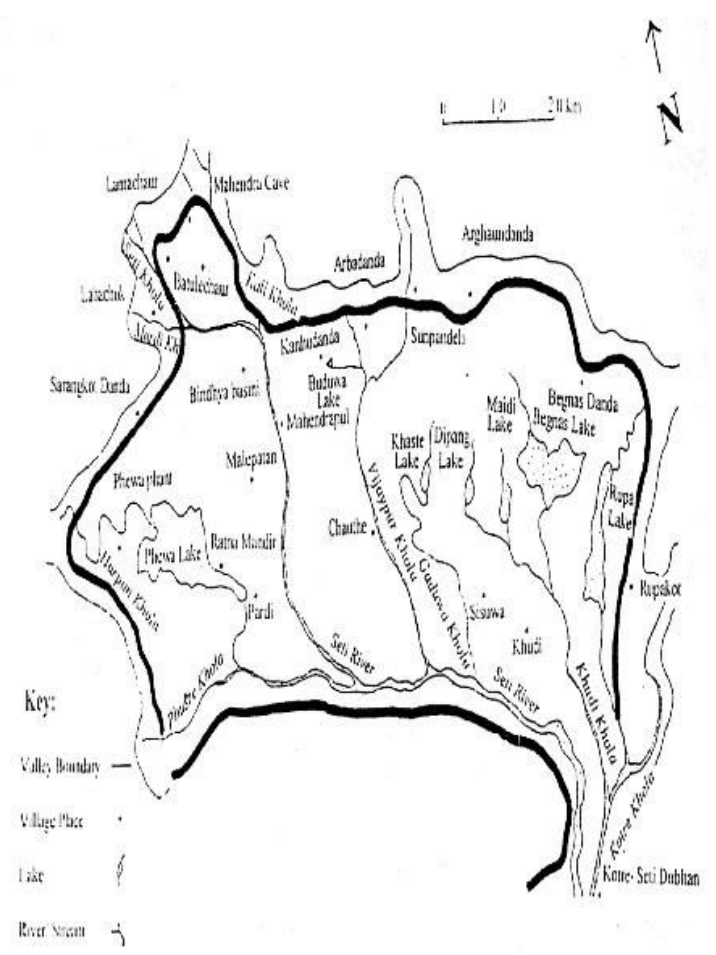

Figure 1. Location map of study area.

\section{Sampling}

The present work was performed during September 2009 to February 2010. Experienced local fishermen were employed and gill nets having different mesh sizes ( 2.5 to $27.5 \mathrm{~cm}$ ) were used for monthly sampling. Fishes were caught, examined and identified on the site and released into water unharmed. The fish samples which required taxonomic verification were collected and preserved in $10 \%$ formalin solution, brought to the laboratory of Department of Zoology, Prithvi Narayan (PN) Campus, TU, Pokhara. The fishes were identified upto species level following Berg (1940), Shrestha (1981, 2001) and Jayaram (1999), and deposited in the museum of the department. 
Information on fish diversity, their habitat condition, conservationissues and aspects related to fisheries were acquired through questionnaire from local people, fisher community, fisheries co-operative committee and field observation. The criteria laid out by IUCN $(1994,2001)$ were followed for assessment of the status of fishes.

\section{RESULTS}

\section{Species diversity and distribution}

The fishes recorded from the lakes viz., Phewa, Begnas, Rupa, Dipang and Maidi are presented in the Table 1. List of fishes shows that 34 species were recorded from these lakes. Lake Rupa was found to have highest number of species (30), followed by Lake Begnas and Lake Phewa (29), Lake Dipang (24) and Lake Maidi (8 species of fishes).

Fish species recorded from all five lakes were Puntius sophore, P. conchonius, Barilius bendelisis, Xenentodon cancila, Channa orientalis, C. punctatus and Mastacembelus armatus. The species recorded from four lakes viz., Phewa, Begnas, Rupa and Dipang were, Neolissocheilus hexagonolepis, Chagunius chagunio, Puntius chola, Tor putitora, Labeo rhita, L. angra, Cirrhinus mrigala, Catla catla, Ctenopharyngodon idella, Cyprinus carpio var communis, C. carpio. var specularis, Hypophthalmichthyes molitrix, Aristichthyes nobilis and Oreochromis niloticus. Likewise, fish species recorded from lakes Begnas, Rupa, Dipang and Maidi was Mystus bleekeri. Those recorded only from three lakes viz., Phewa, Begnas and Rupa were Puntius sarana, Barilius vagra, B.barna and Danio devario. Similarly, the species recorded from lakes Rupa and Dipang was Heteropneustes fossilis.

Some species of exotic fishes viz., Cyprinus carpio var. communis (Common Carp), C. carpio var. specularis (Common Carp),
Hypophthalmichthyes molitrix (Silver Carp), Aristichthyes nobilis (Bighead Carp), Ctenopharyngodon idella (Grass Carp), Oreochromis niloticus (Nile Tilapia) and Clarias gariepinus (African Catfish) were also recorded during the study period.

The status of popular game fish Sahar/Mahaseer, Tor tor, was found to be endangered (EN); Sahar/Mahaseer- Tor putitora, Katle/Copper Mahseer- Neolissocheilus hexagonolepis, Rewa- Chagunius chagunio, and the ZebrafishBrachydanio rerio vulnerable (VU), five species were uncommon (UN) and 24 species common (C) and common exotic( $\left.\mathrm{C}^{*}\right)$.

\section{Occurrence}

Monthly occurrence of different species of fishes recorded during the study period in various lakes are presented in Tables 2-3. The Tor tor , an endangered (EN) species was recorded in low to very low occurrence in four months, vulnerable (VU) species viz., Neolissocheilus hexagonolepis, Chagaunius chagunio, Tor putitora and Brachydanio revio were recorded having low to very low occurrence. Among the uncommon (UN) species Puntius sarana had very low occurrence, Naziritor chelynoides had low to very low occurrence, and Labeo angra and Barilus vagra had moderate to low occurrence. The common (C) species were found to have moderate to high occurrence during all months of the study period. The exotic species Clarias gariepinus and Oreochromis niloticus exceeded almost all other in occurence in most of the lakes. During the study period the indigenous catfish Heteropneustes fossilis was also recorded from Lakes Rupa and Dipang. The fresh water eel (Raj-bam), Anguilla bengalensis, which occurred in considerable number in Lakes Begnas and Rupa could not be recorded during the study period. 
Diversity, distribution and conservation of ...

Table 1. List of fishes and their status in lakes of Pokhara Valley.

\begin{tabular}{|c|c|c|c|c|}
\hline S.N. & Fish Species & $\begin{array}{l}\text { Local/Common } \\
\text { Name }\end{array}$ & Distribution Sites & Status \\
\hline & Family: Cyprinidae & & & \\
\hline 1. & Neolissocheilus hexagonolepis (Mc Clelland) & Katle & $\mathrm{F}, \mathrm{B}, \mathrm{R}, \mathrm{D}$ & VU \\
\hline 2. & Chagunius chagunio (Hamilton - Buchanan) & Rewa & $\mathrm{F}, \mathrm{B}, \mathrm{R}, \mathrm{D}$ & VU \\
\hline 3. & Puntius sophore (Hamilton - Buchanan) & Bhitta & $\mathrm{F}, \mathrm{B}, \mathrm{R}, \mathrm{D}, \mathrm{M}$ & $\mathrm{C}$ \\
\hline 4. & P. sarana (Hamilton - Buchanan) & Kande & $\mathrm{F}, \mathrm{B}, \mathrm{R}$ & $\mathrm{UN}$ \\
\hline 5. & P. chola (Hamilton - Buchanan) & Bhurluk & $\mathrm{F}, \mathrm{B}, \mathrm{R}, \mathrm{D}, \mathrm{M}$ & $\mathrm{C}$ \\
\hline 6. & P. conchonius (Hamilton - Buchanan) & Tike Bhitta & $\mathrm{F}, \mathrm{B}, \mathrm{R}, \mathrm{D}, \mathrm{M}$ & $\mathrm{C}$ \\
\hline 7. & Tor putitora (Hamilton - Buchanan) & Sahar & F,B,R,D & VU \\
\hline 8. & T. tor (Hamilton - Buchanan) & Sahar & $\mathrm{F}$ & $\mathrm{EN}$ \\
\hline 9. & Naziritor chelynoides (Mc Clelland) & Karange & $\mathrm{R}$ & UN \\
\hline 10. & Labeo rohita (Hamilton - Buchanan) & Rohu & F,B,R,D & $\mathrm{C}$ \\
\hline 11. & L. angra (Hamilton - Buchanan) & Gardi & $\mathrm{F}, \mathrm{B}, \mathrm{R}, \mathrm{D}$ & UN \\
\hline 12. & Cirrhinus mirigala (Hamilton - Buchanan) & Naini & $\mathrm{F}, \mathrm{B}, \mathrm{R}, \mathrm{D}$ & $\mathrm{C}$ \\
\hline 13. & C. reba (Hamilton - Buchanan) & Reba & $\mathrm{F}$ & UN \\
\hline 14. & Catla catla (Hamilton - Buchanan) & Bhakur & $\mathrm{F}, \mathrm{B}, \mathrm{R}, \mathrm{D}$ & $\mathrm{C}$ \\
\hline 15. & Ctenopharyngodon idella (Valenciennes) & Grass Carp & $\mathrm{F}, \mathrm{B}, \mathrm{R}, \mathrm{D}$ & $\mathrm{C}^{*}$ \\
\hline 16. & Cyprimus carpio var. communis (Linnaeus) & Scale/ Common cap & F,B,R,D & $\mathrm{C}^{*}$ \\
\hline 17. & C. carpio var. specularis (Linnaeus) & Mirror/Common capp & F,B,R,D & $\mathrm{C}^{*}$ \\
\hline 18. & Hypophthalmichthyes molitrix (Valenciennes) & Silver carp & F,B,R,D & $\mathrm{C}^{*}$ \\
\hline 19. & Aristichthyes nobilis (Richardson) & Big Head Carp & $\mathrm{F}, \mathrm{B}, \mathrm{R}, \mathrm{D}$ & $\mathrm{C}^{*}$ \\
\hline 20. & Barilius bendelisis (Hamilton - Buchanan) & Faketa & $\mathrm{F}, \mathrm{B}, \mathrm{R}, \mathrm{D}, \mathrm{M}$ & $\mathrm{C}$ \\
\hline 21. & B. vagra (Hamilton - Buchanan) & Lam Faketa & $\mathrm{F}, \mathrm{B}, \mathrm{R}$ & UN \\
\hline 22. & B. barna (Hamilton - Buchanan) & Bage Faketa & $\mathrm{F}, \mathrm{B}, \mathrm{R}$ & $\mathrm{C}$ \\
\hline 23. & Danio devario (Hamilton - Buchanan) & Sera/ Silbhitta & $\mathrm{F}, \mathrm{B}, \mathrm{R}$ & $\mathrm{C}$ \\
\hline 24. & Brachydamio rerio (Hamilton - Buchanan) & Zebra fish & B & VU \\
\hline 25. & Esomus danricus (Hamilton - Buchanan) & Sidhra & $\mathrm{B}, \mathrm{R}$ & $\mathrm{C}$ \\
\hline \multicolumn{5}{|c|}{ Family: Bagridae } \\
\hline 26. & Mystus bleekeri (Day) & Junge & $\mathrm{B}, \mathrm{R}, \mathrm{D}, \mathrm{M}$ & $\mathrm{C}$ \\
\hline \multicolumn{5}{|c|}{ Family: Clariidae } \\
\hline 27. & Clarias batrachus (Linnaeus) & Magur & $\mathrm{F}$ & $\mathrm{C}$ \\
\hline 28. & C. gariepimus (Burchell) & Bikasi Magur & $\mathrm{F}, \mathrm{R}, \mathrm{D}$ & $\mathrm{C}^{*}$ \\
\hline \multicolumn{5}{|c|}{ Family: Heteropneustidae } \\
\hline 29. & Heteropneustes fossilis (Bloch) & Balim & R,D & $\mathrm{C}$ \\
\hline \multicolumn{5}{|c|}{ Family: Belonidae } \\
\hline 30. & Xenentodon cancila (Hamilton-Buchanan) & Chuche Bam & F,B,R,D,M & $\mathrm{C}$ \\
\hline \multicolumn{5}{|c|}{ Family: Channidae } \\
\hline 31. & Channa orientalis (Bloch and Schneider) & Bhoti (Hile) & F,B,R,D,M & $\mathrm{C}$ \\
\hline
\end{tabular}


Ganesh Timilsina et al.

\begin{tabular}{|c|c|c|c|c|}
\hline 32. & C. punctatus (Bloch) & Bhot (Hile) & $\mathrm{F}, \mathrm{B}, \mathrm{R}, \mathrm{D}, \mathrm{M}$ & $\mathrm{C}$ \\
\hline \multicolumn{5}{|c|}{ Family: Cichlidae } \\
\hline 33. & Oreochromis niloticus (Linnaeus) & Tilapia & $\mathrm{F}, \mathrm{B}, \mathrm{R}, \mathrm{D}$ & $\mathrm{C}^{*}$ \\
\hline \multicolumn{5}{|c|}{ Family: Mastacembelidae } \\
\hline 34. & Mastacembelus armatus (Lacepede) & Bam & $\mathrm{F}, \mathrm{B}, \mathrm{R}, \mathrm{D}, \mathrm{M}$ & $\mathrm{C}$ \\
\hline
\end{tabular}

\section{Abbreviation:}

a. Sites: $\mathrm{P}=$ Phewa, $\mathrm{B}=$ Begnas, $\mathrm{R}=\mathrm{Rupa}, \mathrm{D}=$ Dipang, $\mathrm{M}=$ Maidi

b. Status: $\mathrm{C}=$ Common, $\mathrm{UN}=$ Uncommon, $\mathrm{EN}=$ Endangered, $\mathrm{VU}=$ Vulnerable, $\mathrm{C}^{*}=$ Common Exotic.

Table 2. Monthly occurrence of fishes in lakes Phewa and Begnas.

\begin{tabular}{|c|c|c|c|c|c|c|c|c|c|c|c|c|c|}
\hline \multirow[t]{3}{*}{ S.N. } & \multirow[t]{3}{*}{ Fish Species } & \multicolumn{12}{|c|}{ Months } \\
\hline & & \multicolumn{2}{|c|}{ Sep. } & \multicolumn{2}{|c|}{ Oct. } & \multicolumn{2}{|c|}{ Nov. } & \multicolumn{2}{|c|}{ Dec. } & \multicolumn{2}{|c|}{ Jan. } & \multicolumn{2}{|c|}{ Feb. } \\
\hline & & $\mathbf{P h}$ & $\mathrm{Be}$ & $\mathrm{Ph}$ & $\mathrm{Be}$ & $\mathrm{Ph}$ & $\mathrm{Be}$ & $\mathrm{Ph}$ & $\mathrm{Be}$ & $\mathrm{Ph}$ & $\mathrm{Be}$ & $\mathrm{Ph}$ & $\mathrm{Be}$ \\
\hline 1. & Neolissocheilus hexagonolepis & c & $\mathrm{b}$ & $\mathrm{b}$ & $\mathrm{b}$ & $\mathrm{b}$ & $\mathrm{b}$ & $\mathrm{b}$ & $\mathrm{b}$ & $\mathrm{a}$ & a & $\mathrm{a}$ & a \\
\hline 2. & Chagunius chagunio & $\mathrm{b}$ & c & $\mathrm{b}$ & $\mathrm{b}$ & $\mathrm{b}$ & $\mathrm{b}$ & $\mathrm{b}$ & $\mathrm{b}$ & a & a & a & a \\
\hline 3. & Puntius sophore & $\mathrm{b}$ & c & d & d & d & d & d & d & d & $d$ & d & $\mathrm{d}$ \\
\hline 4. & P. sarana & b & $\mathrm{a}$ & $\mathrm{a}$ & $\mathrm{a}$ & $\mathrm{a}$ & $\mathrm{a}$ & $\mathrm{a}$ & - & $\mathrm{a}$ & - & $\mathrm{a}$ & - \\
\hline 5. & P. chola & $\mathrm{b}$ & $\mathrm{b}$ & $\mathrm{b}$ & $\mathrm{b}$ & $a$ & a & $\mathrm{a}$ & $\mathrm{a}$ & $a$ & a & $\mathrm{a}$ & a \\
\hline 6. & P. conchonius & $\mathrm{b}$ & $\mathrm{c}$ & $\mathrm{d}$ & $\mathrm{d}$ & $\mathrm{d}$ & $\mathrm{d}$ & $\mathrm{d}$ & $\mathrm{d}$ & $\mathrm{d}$ & d & $\mathrm{d}$ & $\mathrm{d}$ \\
\hline 7. & Tor putitora & $\mathrm{c}$ & $\mathrm{c}$ & $\mathrm{b}$ & $\mathrm{b}$ & $\mathrm{b}$ & $\mathrm{b}$ & $\mathrm{b}$ & $\mathrm{b}$ & $a$ & $\mathrm{a}$ & $\mathrm{a}$ & a \\
\hline 8. & Tor tor & $\mathrm{b}$ & - & $a$ & - & $a$ & - & $\mathrm{a}$ & - & - & - & - & - \\
\hline 9. & Labeo rohita & $\mathrm{b}$ & $\mathrm{b}$ & $\mathrm{b}$ & $\mathrm{b}$ & $\mathrm{b}$ & $\mathrm{b}$ & $\mathrm{b}$ & $\mathrm{b}$ & $\mathrm{b}$ & $\mathrm{b}$ & $\mathrm{b}$ & $\mathrm{b}$ \\
\hline 10. & L. angra & $\mathrm{b}$ & - & $\mathrm{c}$ & - & c & - & $\mathrm{d}$ & $\mathrm{a}$ & $d$ & a & $d$ & a \\
\hline 11. & Cirrhinus mrigala & $\mathrm{b}$ & $\mathrm{b}$ & $\mathrm{b}$ & $\mathrm{b}$ & $\mathrm{b}$ & $\mathrm{b}$ & $\mathrm{b}$ & $\mathrm{b}$ & $\mathrm{b}$ & $\mathrm{b}$ & $\mathrm{b}$ & $\mathrm{b}$ \\
\hline 12. & C. reba & c & - & c & - & c & - & $\mathrm{b}$ & - & $\mathrm{b}$ & - & $\mathrm{b}$ & - \\
\hline 13. & Catla catla & c & c & $\mathrm{d}$ & $d$ & $d$ & $d$ & $d$ & d & $d$ & $\mathrm{~d}$ & d & $\mathrm{d}$ \\
\hline 14. & Ctenopharyngodon idella* & $\mathrm{b}$ & $\mathrm{b}$ & a & $\mathrm{b}$ & $\mathrm{b}$ & $\mathrm{b}$ & $\mathrm{b}$ & $\mathrm{b}$ & $\mathrm{b}$ & $\mathrm{b}$ & $\mathrm{b}$ & $\mathrm{b}$ \\
\hline 15. & Cyprinus carpio var. communis* & $\mathrm{b}$ & $\mathrm{b}$ & $\mathrm{b}$ & $\mathrm{b}$ & $\mathrm{b}$ & $\mathrm{b}$ & $\mathrm{b}$ & $\mathrm{b}$ & $\mathrm{b}$ & $\mathrm{b}$ & $\mathrm{b}$ & b \\
\hline 16. & C.carpio var. specularis* & $\mathrm{b}$ & $\mathrm{b}$ & $\mathrm{b}$ & $\mathrm{b}$ & $\mathrm{b}$ & $\mathrm{b}$ & $\mathrm{b}$ & $\mathrm{b}$ & $\mathrm{b}$ & $\mathrm{b}$ & $\mathrm{b}$ & $\mathrm{b}$ \\
\hline 17. & Hypophthalmichthyes molitrix* & b & $\mathrm{b}$ & $\mathrm{d}$ & $\mathrm{d}$ & $\mathrm{d}$ & $d$ & $d$ & d & $d$ & $\mathrm{~d}$ & $d$ & $\mathrm{~d}$ \\
\hline 18. & Aristichthyes nobilis* & $\mathrm{b}$ & $\mathrm{c}$ & $d$ & $d$ & $d$ & d & $d$ & $\mathrm{~d}$ & $\mathrm{~d}$ & $\mathrm{~d}$ & $d$ & $\mathrm{~d}$ \\
\hline 19. & Barilius bendelisis & $\mathrm{b}$ & $\mathrm{b}$ & $\mathrm{b}$ & $\mathrm{b}$ & $\mathrm{b}$ & b & c & c & $\mathrm{d}$ & c & d & c \\
\hline 20. & B. vagra & $\mathrm{b}$ & $\mathrm{b}$ & $\mathrm{b}$ & $\mathrm{b}$ & $\mathrm{b}$ & $\mathrm{b}$ & $\mathrm{b}$ & $\mathrm{b}$ & $\mathrm{c}$ & $\mathrm{b}$ & c & c \\
\hline 21. & B. barna & c & $\mathrm{b}$ & c & $\mathrm{b}$ & c & $\mathrm{b}$ & c & $\mathrm{b}$ & $\mathrm{c}$ & a & $\mathrm{b}$ & a \\
\hline 22. & Damio devario & $\mathrm{b}$ & $\mathrm{b}$ & $\mathrm{b}$ & $\mathrm{b}$ & $\mathrm{b}$ & $\mathrm{b}$ & $\mathrm{b}$ & $\mathrm{b}$ & c & c & c & $\mathrm{C}$ \\
\hline 23. & Brachydanio rerio & - & - & - & - & - & - & - & $\mathrm{a}$ & - & a & - & $\mathrm{a}$ \\
\hline 24. & Esomms danricus & - & - & - & - & - & $\mathrm{a}$ & - & a & - & a & - & a \\
\hline
\end{tabular}


Diversity, distribution and conservation of ...

\begin{tabular}{|l|l|l|l|l|l|l|l|l|l|l|l|l|l|}
\hline 25. & Mystus bleekeri & - & b & - & b & - & b & - & a & - & a & - & a \\
\hline 26. & Clarias batrachus & c & - & c & - & c & - & b & - & b & - & b & - \\
\hline 27. & C. gariepimi* & b & - & b & - & b & - & b & - & a & - & a & - \\
\hline 28. & Xenentodon cancila & b & b & b & b & b & b & b & b & a & a & a & a \\
\hline 29. & Channa orientalis & c & b & c & c & b & c & b & b & b & b & b & b \\
\hline 30. & C. punctatus & c & c & c & c & b & c & b & b & b & b & b & b \\
\hline 31. & Oreochromis niloticus* & b & d & b & d & b & d & b & d & b & d & b & d \\
\hline 32. & Mastacembelus armatus & b & c & b & c & b & b & b & b & a & b & a & b \\
\hline
\end{tabular}

\section{Occurrence rank and sites:}

$(-)=$ absent,$a=$ very low occurrence, $b=$ low occurrence, $c=$ moderate occurrence, $d=$ high occurrence, $\mathrm{Ph}=$ Phewa, $\mathrm{Be}=$ Begnas

Table 3. Monthly occurrence of fishes in lakes Rupa, Dipang and Maidi

\begin{tabular}{|c|c|c|c|c|c|c|c|c|c|c|c|c|c|c|c|c|c|c|c|}
\hline \multirow{3}{*}{ S.N. } & \multirow{3}{*}{ Finh Spexies } & \multicolumn{18}{|c|}{ Monthg } \\
\hline & & \multicolumn{3}{|c|}{ Sep. } & \multicolumn{3}{|c|}{ Oct. } & \multicolumn{3}{|c|}{ Nav. } & \multicolumn{3}{|c|}{ Dec. } & \multicolumn{3}{|c|}{ Jan. } & \multicolumn{3}{|c|}{ Feh. } \\
\hline & & $\mathbf{R u}$ & $\mathbf{D i}$ & Mn & $\mathbf{R u}$ & $\mathbf{D i}$ & $\mathbf{M a}$ & Ru & $\overline{D_{i}}$ & Mr & $\mathbf{R u}$ & $\mathbf{D i}$ & $\mathrm{Ma}$ & Rin & $\mathbf{D i}$ & Mn & $\mathbf{R u}$ & $\mathbf{D i}$ & $\mathrm{Ma}$ \\
\hline 1. & Nealissocheilus hexagonolepis & b & $\mathrm{b}$ & - & $\mathrm{b}$ & $\mathrm{b}$ & - & $\mathrm{b}$ & $\mathrm{b}$ & - & a & a & - & a & $\mathrm{a}$ & - & $\mathrm{a}$ & a & - \\
\hline 2. & Chagunius chagumio & $\bar{b}$ & $\mathrm{~b}$ & - & $\mathrm{b}$ & $\mathrm{b}$ & - & a & $\mathrm{b}$ & - & a & $\bar{a}$ & - & - & $\bar{a}$ & - & - & $\bar{a}$ & - \\
\hline 3. & Puntius sophore & c & $\mathrm{c}$ & c & $\mathrm{c}$ & c & - & $\mathrm{c}$ & $\mathrm{c}$ & - & $\mathrm{c}$ & $\mathrm{c}$ & - & c & c & - & c & c & - \\
\hline 4. & P. sarama & a & $a$ & - & $\mathrm{a}$ & $\mathrm{a}$ & c & $\mathrm{a}$ & - & $\mathrm{c}$ & - & - & $\mathrm{c}$ & - & - & c & - & - & $\mathrm{c}$ \\
\hline 5. & P. chola & $\mathrm{b}$ & $a$ & - & $\mathrm{b}$ & $a$ & - & $\mathrm{b}$ & $\mathrm{a}$ & - & $\mathrm{a}$ & $\mathrm{a}$ & - & a & - & - & $\mathrm{a}$ & - & - \\
\hline 6. & P. conchonius & b & $\mathrm{c}$ & $\mathrm{c}$ & $\mathrm{b}$ & $\mathrm{c}$ & $\mathrm{c}$ & $\mathrm{b}$ & $\mathrm{c}$ & $\mathrm{c}$ & $\mathrm{b}$ & $\mathrm{b}$ & $\mathrm{b}$ & $\mathrm{b}$ & b & b & $\mathrm{b}$ & $\mathrm{b}$ & $\mathrm{b}$ \\
\hline 7. & Tor putitora & $\mathrm{b}$ & $\mathrm{b}$ & - & $\mathrm{b}$ & $a$ & - & $\mathrm{b}$ & $a$ & - & a & - & - & a & - & - & a & - & - \\
\hline 8. & Naziritor chelynoides & b & - & - & $\mathrm{b}$ & - & - & $\mathrm{b}$ & - & - & $\mathrm{b}$ & - & - & $\mathrm{a}$ & - & - & $\mathrm{a}$ & - & - \\
\hline 9. & Labeo rohita & - & - & - & - & - & - & - & $\mathrm{b}$ & - & - & a & - & a & a & - & a & a & - \\
\hline 10. & L. angra & - & - & - & - & - & - & - & $\mathrm{b}$ & - & - & $\mathrm{a}$ & - & a & $\mathrm{a}$ & - & $\mathrm{a}$ & $\mathrm{a}$ & - \\
\hline 11. & Cirrhimus mrigala & b & $\mathrm{b}$ & - & $\mathrm{b}$ & $\mathrm{b}$ & - & $\mathrm{b}$ & $\mathrm{b}$ & - & $\mathrm{a}$ & $\mathrm{b}$ & - & $\mathrm{b}$ & $\mathrm{b}$ & - & $\mathrm{b}$ & $\mathrm{b}$ & - \\
\hline 12. & Catla catla & $\mathrm{c}$ & $\mathrm{b}$ & - & $\bar{c}$ & $\mathrm{~b}$ & - & $\mathrm{d}$ & $\mathrm{c}$ & - & d & $\bar{c}$ & - & d & $\mathrm{c}$ & - & d & $\mathrm{c}$ & - \\
\hline 13. & $\begin{array}{l}\text { Ctenopharyngodon } \\
\text { idella* }\end{array}$ & c & $\mathrm{c}$ & - & $\mathrm{b}$ & c & - & $\mathrm{b}$ & $\mathrm{b}$ & - & $\mathrm{b}$ & $\mathrm{b}$ & - & b & b & - & $\mathrm{b}$ & $\mathrm{b}$ & - \\
\hline 14. & $\begin{array}{l}\text { Cyprimus carpio var. } \\
\text { commminis* }\end{array}$ & b & $\mathrm{b}$ & - & $\mathrm{b}$ & $\mathrm{b}$ & - & $\mathrm{b}$ & $\mathrm{b}$ & - & $\mathrm{b}$ & $\mathrm{b}$ & - & $\mathrm{b}$ & b & - & $\mathrm{b}$ & $\mathrm{b}$ & - \\
\hline 15. & C. carpio var. specularis* & $\mathrm{b}$ & $\mathrm{b}$ & - & $\mathrm{b}$ & $\mathrm{b}$ & - & $\mathrm{b}$ & $\mathrm{b}$ & - & $\mathrm{b}$ & $\mathrm{B}$ & - & $\mathrm{b}$ & $\mathrm{b}$ & - & $\mathrm{b}$ & $\mathrm{b}$ & - \\
\hline 16. & $\begin{array}{l}\text { Hypophthalmichthyes } \\
\text { molitrix* }\end{array}$ & c & c & - & $d$ & $\mathrm{c}$ & - & d & $\mathrm{c}$ & - & $\mathrm{b}$ & $\mathrm{c}$ & - & d & c & - & $d$ & c & - \\
\hline 17. & Aristichthyes nobilis* & $\mathrm{c}$ & $\mathrm{c}$ & - & d & d & - & $\mathrm{d}$ & d & - & d & d & - & d & $\mathrm{d}$ & - & d & d & - \\
\hline 18. & Barilius bendelisis & $\mathrm{b}$ & $\mathrm{b}$ & $\mathrm{b}$ & $\mathrm{b}$ & $\mathrm{b}$ & $\mathrm{b}$ & $\mathrm{b}$ & $\mathrm{b}$ & $\mathrm{b}$ & $\mathrm{c}$ & $\mathrm{c}$ & $\mathrm{c}$ & $\mathrm{c}$ & $\bar{c}$ & $\bar{c}$ & $\bar{c}$ & $\bar{c}$ & $\bar{c}$ \\
\hline 19. & B. vagra & $\mathrm{b}$ & $\mathrm{b}$ & b & $\mathrm{b}$ & $\mathrm{b}$ & $\mathrm{b}$ & $\mathrm{b}$ & $\mathrm{b}$ & $\mathrm{b}$ & $\mathrm{b}$ & $\mathrm{b}$ & $\mathrm{a}$ & $\mathrm{c}$ & c & a & $\mathrm{c}$ & $\mathrm{c}$ & $\mathrm{a}$ \\
\hline 20. & B. barna & $\mathrm{b}$ & - & - & $\mathrm{b}$ & - & - & $\mathrm{b}$ & - & - & $\mathrm{b}$ & - & - & $\mathrm{c}$ & - & - & $\mathrm{c}$ & - & - \\
\hline 21. & Damio devario & $\mathrm{b}$ & - & - & $\mathrm{b}$ & - & - & $\mathrm{b}$ & - & - & $\mathrm{c}$ & - & - & $\mathrm{c}$ & - & - & $\mathrm{c}$ & - & - \\
\hline 22. & Esommus danricus & - & - & - & - & - & - & - & - & - & $\mathrm{a}$ & - & - & a & - & - & a & - & - \\
\hline 23. & Mystus bleekeri & $\mathrm{b}$ & b & - & $\mathrm{b}$ & $\mathrm{b}$ & - & b & $\mathrm{b}$ & - & $\mathrm{b}$ & $\mathrm{b}$ & - & a & a & - & $\mathrm{a}$ & a & - \\
\hline
\end{tabular}


Ganesh Timilsina et al.

\begin{tabular}{|l|l|l|l|l|l|l|l|l|l|l|l|l|l|l|l|l|l|l|l|}
\hline 24. & Clarias gariepimis & b & b & - & b & b & - & b & b & - & b & a & - & a & a & - & a & a & - \\
\hline 25. & Heteropneustes fossilis & b & b & - & b & b & - & b & b & - & a & a & - & a & a & - & a & a & - \\
\hline 26. & Xenentodon cancila & c & b & a & b & b & a & b & b & a & b & b & - & a & a & - & a & a & - \\
\hline 27. & Channa orientalis & c & c & b & c & c & b & c & b & b & b & b & a & a & b & a & a & b & a \\
\hline 28. & C. punctatus & c & c & b & c & c & b & c & b & a & b & b & a & a & b & a & a & b & a \\
\hline 29. & Oreochromis niloticus $*$ & b & c & - & b & c & - & c & b & - & b & b & - & b & b & - & b & b & - \\
\hline 30. & Mastacembehis armatus & c & b & b & c & b & b & c & b & b & d & b & a & b & a & a & b & a & a \\
\hline
\end{tabular}

\section{Occurrence rank and sites:}

$(-)=$ absent, $a=$ very low occurrence, $b=$ low occurrence, $\mathrm{c}=$ moderate occurrence, $\mathrm{d}=$ high occurrence, $\mathrm{Ru}=$ Rupa, $\mathrm{Di}=$ Dipang, $\mathrm{Ma}=$ Maidi

\section{Human activities and conservation of aquatic bioresources}

Protection of habitat is the most important aspect to protect the biodiversity in any ecosystem. The natural healthy abiotic condition in the waterbodies is essential for the conservation and protection of the existing fish diversity along with other aquatic bioresources. Various human activities such as direct disposal of domestic sewage, unscientific agricultural practices, construction of roads and buildings, deforestation, soil erosion etc. near and around the lakes were mainly responsible for the degradation of the aquatic habitat of lakes. The encroachment of the shoreline for agricultural practice, waste disposal, animal grazing, construction of dams without fish-ladder or fish-passes, improper fishing practices etc. were creating threats to aquatic ecosystem. The introduction of alien species for higher production is one of the major cause for decrease in productivity of the indigenous species which may lead to remarkable decline in abundance and species richness in the future.

\section{DISCUSSION}

Ferrow and Badgami (1980) mentioned 22 species of fishes from lakes in Pokhara Valley including Tor, Neolissocheilus, Puntius,
Chagunius, Barilius etc. John and Dhewajoo (1989) reported fishes from Begnaslake including Tor, Neolissocheilus, Chagunius, etc. Poudel (1998) observed five species of fishes from lake Satrahazar in Chitwan district including Puntius sophore, Danio devario, Chagunius chagunio, Barilius bende lisis and noted Puntius sophore as dominant species constituting $41.50 \%$ of total fish catch. Pokharel (1999a) reported 25 species from different lakes viz., Phewa, Begnas, Rupa, Dipang, Maidi, Khaste, Gunde, Neureni and Kamal Pokhari including one endangered (EN) species Sahar (Tor tor), four vulnerable species, Sahar (Tor putitora), Katle (Neolissocheilus hexagonolepis), Rewa (Chagunius chagunio) and Zebra Fish (Brachydanio rerio). Bista et al. (2003) recorded 23 species including the important game fish Sahar (Tor putitora) and Katle (Neolissocheilus hexagonolepis), from Lake Rupa. In the present study, 34 species of fishes were recorded from the major five lakes including one endangered (EN) species Sahar (Tor tor), four vulnerable (VU) species viz., Sahar (Tor putitora), Katle (Neolissocheilus hexagonolepis), Rewa (Chagunius chagunio) and Zebra fish (Brachydanio rerio).

Swar and Shrestha (1997) observed that "in the last few decades, inland waters have been subjected to a range of stress caused by direct and indirect human activities such as irrigation, hydroelectric projects, urbanization, industrialization, modernization of agriculture and changes in land use in the river floodplains, thus created adverse effects on aquatic 
Diversity, distribution and conservation of ...

biodiversity especially the native fish fauna. The direct disposal of sewage, mixing of chemical fertilizer and pesticides from nearby agriculture field and deposition of silt due to landslide, soil erosion etc. deteriorate the water quality due to their lethal effects, which kill organisms, have drastic impact on discharge and morphology, increase organic load having high oxygen demand reducing the oxygen supply also reduces the abundance and species richness due to pressure upon food organisms available (Gratwick et al., 2003). Construction of dams were found to cause habitat degradation with adverse effects on biota, as well as alteration in ecology and preventing the migration of fishes which move upstream and downstream for various purposes including breeding, create habitat damage below the dams with alteration in stream flow regime (Bemacseck, 2000; Menon et al., 2000; Pokharel, 2010a).

During the study period the lentic water bodies in Pokhara Valley were found influenced by similar human activities such as deforestation causing landslide, soil erosion and siltation, direct disposal of domestic sewage, washing of agricultural pesticides and fertilizers etc. which probably altered the aquatic habitat and status of the fish diversity.

\section{CONCLUSION}

The increasing anthropogenic activities on the water bodies and their surroundings are mainly responsible for the degraded condition of the aquatic habitat and declining bioresources. Deforestation, landslide, soil erosion, direct disposal of waste, mixing of chemical fertilizer and pesticides from the adjoining agricultural fields, construction of dams obstructing free movement of the fishes etc. were observed as threats for the longevity of the lakes along with diversity and abundance of the fishes. An integrated development plan should be designed for the aquatic habitat management alongwith the watershed area which in turn can play an effective role for management of lakes and of their bioresources.

\section{ACKNOWLEDGEMENTS}

The authors are grateful to Nepal Academy of Science and Technology (NAST), Lalitpur for providing research grant and to Associate Prof. R.G. Dhewajoo, Head, Department of Zoology, P.N. Campus for providing laboratory facilities and suggestions. We are thankful to B.R. Pahari, Mr. H.P. Pokharel and Mr. M. Khadka, Laboratory Staff of Department of Zoology and Botany, P.N. Campus for their assistance in field and laboratory works.

\section{REFERENCES}

Agriculture Research Center (ARC). Pokhara (2001-02). Annual Report. Agriculture Research Centre (Fisheries), Pokhara.

Berg, L. S. (1940). Classification of Fishes both Living and Fossils. Edward Brothers Inc, Ann Arbor, Michigan.

Bernacseck, G. (2000). Environmental Issues, Capacity and Information Base for Management of Dam Fisheries. A report submitted to World Commission on Dams (dams report. org).

Bista, J. D., R. P. Dhakal and T. B. Gurung (2003). Changing environmental status and its impacts on flora and fauna of Phewa and Rupa Lakes of Pokhara Valley, Nepal. Proceedings of International Seminar on Mountains-Kathmandu (March 6-8, 2002). Royal Nepal Academy of Science and 
Technology (RONAST), Kathmandu.

Ferrow, W. and P. R. Badagami (1980). On the biology of the commercially important species of fish of the Pokhara Valley, Nepal. Journal of Institute of Science. 3(1): 237-250.

Gratwicke B., B. E. Marshall and N. Hiwatida (2003). The distribution and relative abundance of stream fishes in the upper Manyame River, Zimbabwe, in relation to land use, pollution and exotic predators. African Journal of Aquatic Science 28(1): 25-34.

IUCN (1994). IUCN Red List Categories. International Union for the Conservation of Nature and Natural Resources (IUCN), Gland, Switzerland.

IUCN (2001). IUCN Red List Categories Criteria (Version 3.1). International Union for Conservation of Nature and Natural Resources (IUCN), Gland, Switzerland.

Jayaram, K. C. (1999). The Freshwater Fishes of Indian Region. Narendra Publishing House, New Delhi, India.

John, A. and R. G. Dhewajoo (1989). Effects of Ecological Parameters on Fishes of Pokhara Valley. Report submitted to Royal Nepal Academy of Science and Technology (RONAST), Kathmandu.

Menon A. G. K., H. R. Singh and N. Kumar (2000). Present eco-status of cold water fish and fisheries. In: Cold Water Aquaculture and Fisheries. Singh, H.R. and W.S. Lakra (eds.), Narendra Publishing House New Delhi, 1-36 pp.

Pokharel, K. K. (1999a). Fish biodiversity of lakes in Pokhara Valley. Proceedings of Third National Conference on Science and Technology, Volume II. Royal Nepal Academy of Science and Technology (RONAST), Kathmandu, Nepal, 920-950 pp.
Pokharel, K. K. (2010a). Fish diversity of Seti Gandaki River and Vijayapur Stream and need for their conservation Zoo Journal 1: 40-50.

Pokharel, K. K., G. Timilsina, S. C. Bastola and S. Gurung (2010). Studies on Fisheries Resources, Zoobenthos, Some Abiotic Parameters and Socio-economic Aspects of Fishermen Involved in Lakes of Pokhara Valley. Report submitted to Nepal Academy of Science and Technology (NAST), Lalitpur.

Shrestha, J. (1981). Fishes of Nepal. Curriculum Development Centre, Tribhuvan University, Kathmandu, Nepal.

Shrestha, J. (2001). Taxonomic revision of fishes of Nepal. In: Environment and Agriculture Bio-diversity, Agriculture and Pollution in South Asia. Jha P. K. et al. (eds.), Ecological Society (ECOS), Kathmandu, Nepal, 171-180 pp.

Swar, D. B. and J. Shrestha (1997). Human impact on aquatic ecosystems and native fishes of Nepal. In: Proceedings of the National Symposium on the Role of Fisheries and Agriculture in the Economic Development of Rural Nepal. Nepal Fisheries Society (NEFIS), Kathmandu.

Tripathi, M. P. (1984-85). Ecology of Pokhara Valley. In: Nepal-Natures Paradise, T.C. Majupuria (edi.). White Lotus Company, Bangkok, Thailand, 438-452 pp.

(Received July 5, 2019; revised accepted September $8,2019)$ 